\title{
SIMULATION OF A GAS TURBINE ENGINE ON ZYNQ EMBEDDED PLATFORM
}

\author{
RAKSHITHA S \& SHARMILA K P
}

Department of Electronics and Communication, CMR Institute of Technology, Bengaluru-560037

\begin{abstract}
The digital control features are implemented in designing modeling of an engine where time consumed to test an engine model is reduced. To basically move an aircraft via the air and to produce thrust, we have to employ some type of propulsion system and the most extensively utilized form of propulsion system for modern airplane is gas turbine engine. This paper introduces virtual engine model on ZYNQ embedded platform with all the functions of an actual as turbine engine and it's connected to ECU for simulation process, where it generates real-time work environment parameters for testing and debugging. The input data fed to the model is fuel through on-board 12 bit XADC channel, providing PLA data sets to the INLET from external supply unit. Since there are numerous advancements in pipeline to improve the efficiency and performance of gas turbine engines, we can implement it by integrating all the individual code blocks into a single engine model and analyse the results. This improves the efficiency of the program to run the engine model within the given time by considering real-time parameters. Therefore debug-test-verify period will be optimised making way for advanced and robust thrust providers with maximum control features along with safety standards.

KEYWORDS: Gas Turbine Engine, ZYNQ, XADC, FADEC, ECU, EEC
\end{abstract}

Received: Jun 08, 2020; Accepted: Jun 28, 2020; Published: Sep 08, 2020; Paper Id.: IJMPERDJUN20201123

\section{INTRODUCTION}

In general gas turbine engine comprise of five prime segments via an inlet, combustion, a compressor, an exhaust and the turbine. These engines drive on the principle of Brayton cycle. Brayton cycle is thermodynamic closed cycle of turbine engines where the gas or liquid go through a closed loop, and where working gas that is air compresses it by a compressor as the working fluid such as gas and hence heated by combustion energy of the fuel at the initial and burned-out below persistent pressure state. The internal combustion of engine utilizes air as working fluid where the engine tries to extract the chemical energy from fuel and transforms to mechanical energy to run engine and propel aero-plane. Motive of these turbine engines governs the design in such an extent where cleave of energy betwixt thrust and shaft work is attained. These turbine engines are turbo shaft power plants which permit enormous power and could be used in diversity of applications. The energy is generated by igniting the fuel air mixture in engine and accelerating the gas enormously. These high velocity gasoline are directed via turbine wheels which change the axial movement of the gas to rotary motion. Rotary power now would drive power shaft, then drives a rotor transmission or propeller. Unique prime for's of gas turbines is their fuel pliability. Could be customized or may be adapted to make use of practically some of flammable gas and or light distillate petroleum products like petrol, paraffin, and diesel which occur to be accessible locally, though natural gas is most often used fuel. The gas turbine utilizes three unlike varieties of fuels in combustion chamber such as liquid petroleum or hydrocarbon fuels, gaseous fuels and solid fuels. 
This paper aims at simulation of a gas turbine engine on an embedded platform. Fully Authorized Digital Electronic Control (FADEC) consists of digital computer called as ECU or EEC (Electronic Engine Controller). For provided flight condition FADEC aim is to give optimum engine efficiency. ECU is used to control various parameters of gas turbine engine and performance of an engine. Practically it's hard \& tedious to generate all real-time work environment parameters for testing \& debugging. This problem is addressed or overcome by creating \& deploying an engine model on a Xilinx Zynq embedded platform that is by creating a virtual engine model and this model can be connected with ECU for simulation process.

\section{PROPOSED APPROACH}

We know that engines are the main thrust providers for the aircraft, it makes aircraft to move and as well as fly and numerous advancements in pipeline to improve the efficiency and performance of turbine engines. Hence this puts forth the need for the digital copy of engine.

The Engine model is developed on the embedded hardware platform using the Xilinx Vivado and the Xilinx SDK is deployed on the target FPGA board. This reduces the time consumed to test an engine model, where digital control features are implemented in designing modeling of an engine. Also the debug tests verify is optimized. Proposed schematic engine model is shown in fig. 1 below.

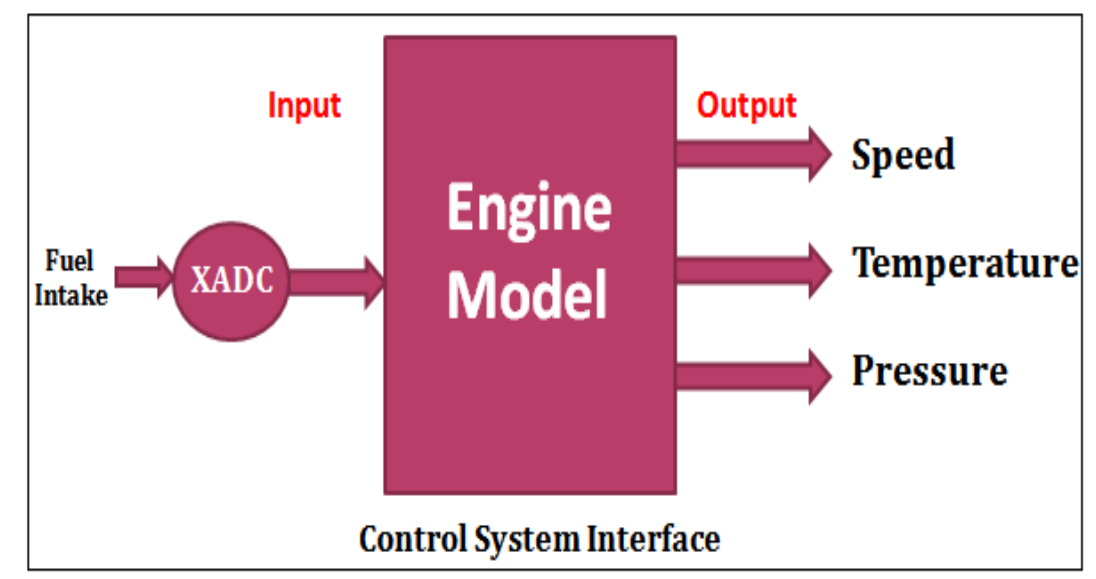

Figure 1: Proposed Structure of Engine Model.

From above figure the primary input fed to system model is fuel which ranges from 0.16 to 1.0 , where number of inputs includes 60001 parameters through via fuel intake. This fuel prior to engine model is fed to XADC channel, providing PLA data sets to the INLET from external supply unit i.e., user throttle requirement. XADC to PS interface permit to monitor the capability of reliable system without the need of PL. The transmission or communication betwixt XADC block and PS occur via JTAG interface. The interface selected is an AXI4Lite, it enables to integrate i.e., to monitor and address large range of analog data acquisition by flexible ADC converter with PL. The channel sequencer is selected as the XADC operating mode and timing mode of XADC is continues as it samples and converts the selected channels. The DCLK frequency is enabled in the range of $100 \mathrm{MHZ}$ and channel averaging by 16 . The XADCIF clock frequency is the PCAP-2x clock / [TCLKRATE] where [TCLKRATE] bit is divided by default 4.Therefore the data output results obtained that is where speed, temperature and pressure is next further transferred on model to control centre where the overall efficiency mapping of the engine model can be attained. 


\section{DEVELOPMENT ENVIRONMENT}

We know the Gas turbine engine consists of many stages. The function of each stage is significantly important. It has to take care all of the parameters like intake of fuel, temperature, pressure, speed and so forth. All these stages have to be integrated into a single model. The execution time of the engine model has to be scaled down within 1 milli-second. To integrate all the stages of the gas turbine engine into a single model a good platform is required. The platform which has good interface between the hardware and software is considered that is Zed Board is chosen as the development platform.

The model of these gas turbine engines is deployed on Xilinx Zynq FPGA platform where engine model is run on the Zynq processor to validate its performance and quality. ZEDis a Zynq7000 all programmable SoC based kit which integrates a dual-core advanced RISC machine cortex application9 processor with FPGA logic.This board has an on or an off switch which is SW8 (pin 8) to gate 12volt supply. The PL of Zynq is equivalent to that of an FPGA. The PS IP wrapper are represented betwixt processor and logic as a logic connection where further can be integrated to custom and embedded IPs with PS.

\section{SYSTEM IMPLEMENTATION}

The complete working process of the gaseous turbine engine is divided into multiple stages based on the actual engine process flow. The same grading method is employed to develop embedded code functions using the eclipse IDE of Xilinx SDK as per the engine functionality. The coding platform in Eclipse IDE is cross-compilation suitable which supports code development. However, C programming language is selected for the development of code.

IP building and connection stages serve as the initial step in building an engine model using Vivado Xilinx Design Suite. An IP is GUI which enables a rapid connection of IP by CUI. As AXI interconnect interface is a point to point interconnect protocol it constitutes into successive levels or layers of block design which comprises of multiple logic core IP's where the configuration and connection is established in system design. It let's any combination of interface connected to master and slave devices. Furthermore design is enabled with auto connect feature as shown in block design of engine model of figure 2, that is it runs connection automation to interconnect all the IP blocks initialized in the design. This also enables users to accelerate the design productivity by validating the design, checking for errors in IP and connections inturn. The validated designs are then wrapped, and this generates a high-level hardware description language file for sub system (IP integrator). Next the data is made to run automatically permitting continual run attempts which varies with RTL-versions, timing analysis, synthesis i.e., is made to run to generate hardware description language net-list generation file and implementation. As a final step the hardware design is exported to SDK platform by generating the bit-stream i.e., all the final outputs which were required for programming on FPGA is launched which means building a hardware block image is completed. 


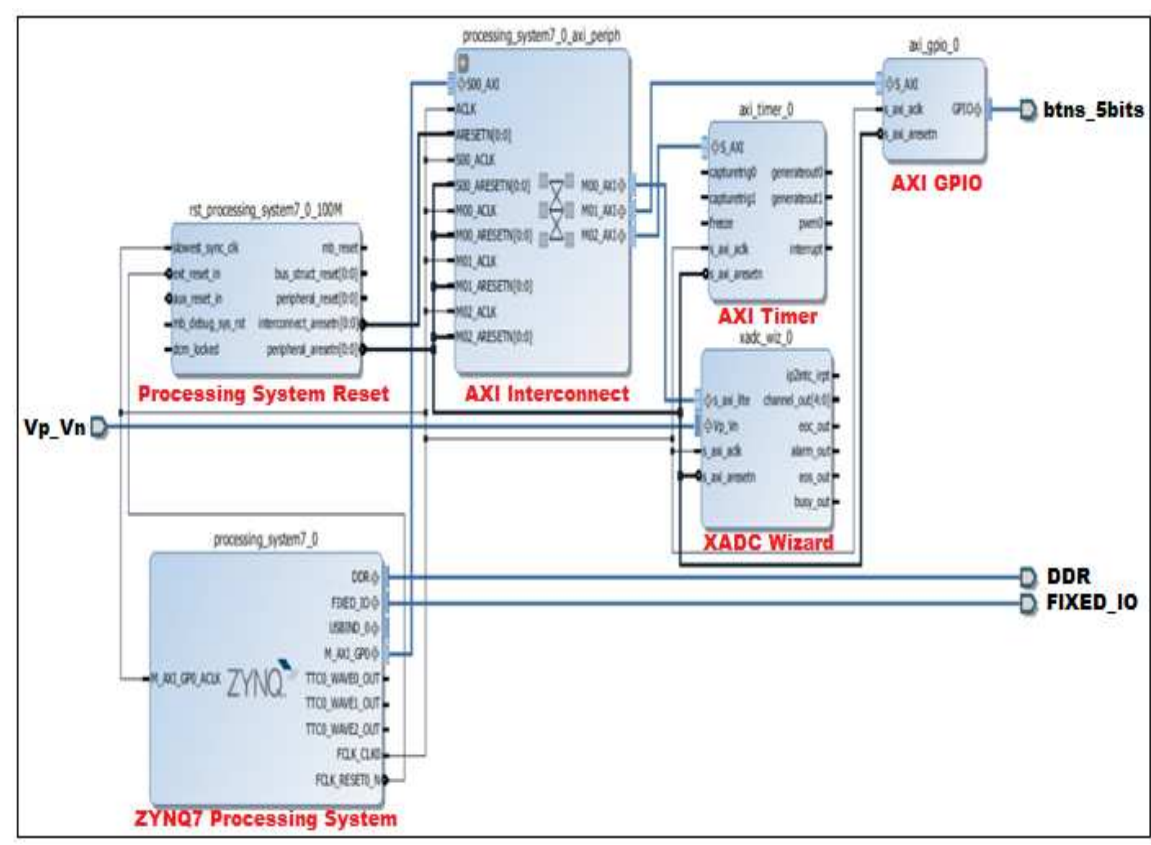

Figure 2: Block Design of Engine Model in Vivado.

Post development of the sketch in the SDK platform, the code is compiled to check for error identification and debugging. This platform includes driver support and certain tools for debugging and profiling and so on. This step serves as the penultimate step before the code is put on the target hardware for result analysis. COM port is set based on USBUART connection given to PC and this is established for communication purpose and before COM port is selected, board is powered on which indicates a green power LED is on. Next in order to download bit-stream to PL, we need to program FPGA. Hence the baud rate is set to 115200,8 data bits and 1 stop bit is selected. The code is analyzed by the system debugger (GDB) for fatal errors before the code is burnt to the SoC on the Zynq board. SDK is also equipped with the efficient debugging algorithms that make the SDK best suite for procedural process. As a result, the engine run values are printed on SDK terminal window and finally efficiency of the model and target set is achieved.

Hence the process of design and development of gasoline turbine engine on Zynq FPGA is done, where all the parameters are aligned to the requirements. Communication protocols are enabled, communication channels connected and baud rate matching is performed for transfer of program from the computer to the target hardware. Data monitoring and performance analysis feature is enabled through the serial communication protocol which provides an option to view the process running on the target board and continuous monitoring of the interim results. Therefore engine model running on the FPGA board is continuously monitored for the checking and validating the results at individual functions right from the beginning of the engine marked by the fuel intake stage which accepts air intake data from the XADC (PLA to be in the actual engine) to the final stage signified by the Nozzle or the exhaust which provides enough thrust for the aircraft for its propulsion.

\section{RESULTS}

We know the given target execution time of an engine model to be run within 1 milli second. This is achieved from below plot results where the overall execution time after simulating an engine model in every stage of engine results in approximated to 0.2 milli seconds as shown in below figure 3. The execution time of Intel i5 / Windows is found to be 10 seconds. The breakthrough results in execution time less than $80 \%$ of the target time. The level of resonance of the engine 
model with the actual gas turbine engine by considering the real-time engine run conditions and parameters within considerable run time is much lesser than the actual engine and it's the major break-through.

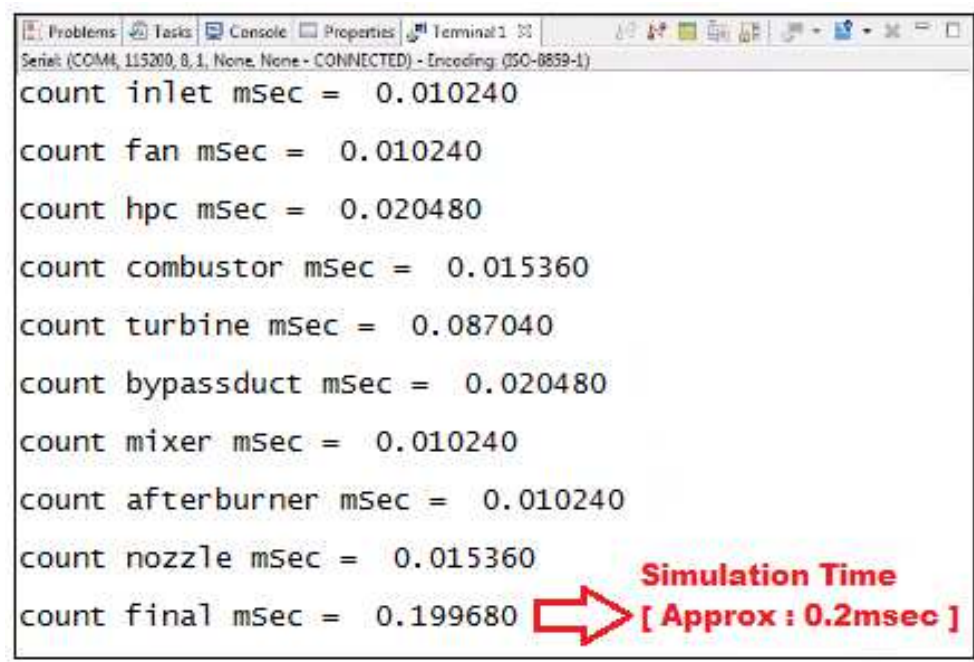

Figure 3: Simulation Results of all Stages of Engine Model.

Data thus collected by engine run is plotted against various parameters to validate its performance such as temperature, pressure and speed. The following graphical representations serve as the proof for the results obtained.

The results obtained by running the developed engine model on the Zynq processor has been plotted on the MATLAB computing environment to verify the resemblance of the obtained results with the actual engine run properties. The below figure 4 shows the relation between the fuel intake input values and the time.

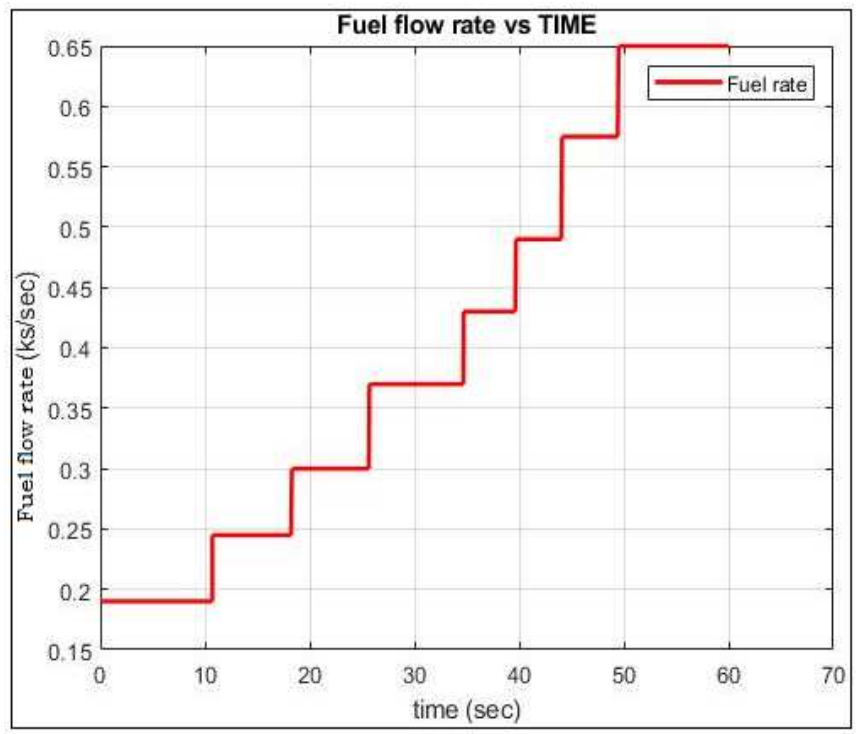

Figure 4: Engine Fuel flow Rate vs Time Plot.

Fuel intake is fed into the GTO for engine performance. The graph shows the increment of GTO value with respect to change in PLA at regular intervals of time during the engine run. Hence the above provided graph signifies the resonance between the obtained results and the actual results.Henceforth the values of gas turbine engine oil plotted against and time based on the results obtained provides a real-time similarity with the actual gas turbine engine. 
The relation between the temperature and time is shown in figure 5. Temperature has a significant role in engine. Higher the temperature permits to improve the efficiency of engine and also increases power in turn. Also we know that performance of engine is dependent on mass of air which is entering engine. Depending on the engine model architecture, engine utilizes or experience large or high temperature gradients, where normal operation of these engines results in temperature that varies at air inlet to 1000 degree Celsius or more in turbine part as reflected in figure.

The below graph plot is plotted for temperature T3 (turbine temperature) which is generated at the Turbine stage against time and temperature T5 (Mixer temperature), being generated at the mixer stage against time.

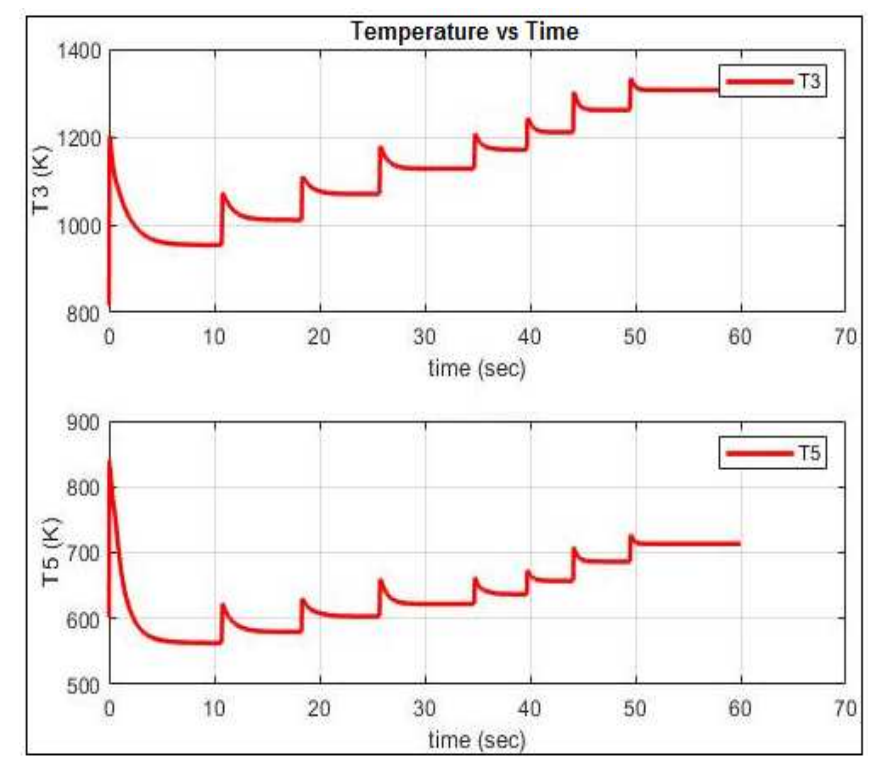

Figure 5: Temperature vs Time Plot.

Thus the relation between temperature and fuel intake to be sustained at various thrust level requirements that is the air intake from the fuel intake stage is compressed at the compressor stage to very high temperature by balancing the pressure. This very high temperature at high pressure level ignites the fuel at later stages. This temperature should be well maintained in the engine run period to sustain this engine at various thrust level requirements.

Pressure is key enabler in working of these turbine engines. In general rise in ratio of pressure is one of the straight way to rise thermal efficiency. The highest pressure is either at end of compressor stage or it can be limited to right before power turbine. Also pressure plays a vital role in maintaining the velocity of the thrust air produced and the temperature at which the fuel is burnt. The graph figure 6 shows the variation in the pressure at different fuel input values with respect to time.

When usually air starts penetrating into these engines aerodynamic and as well energy demand for transformation in pressure and velocity of air. Hence basically throughout the compression stage increase in velocity isn't needed but increase in air pressure is needed or required. 


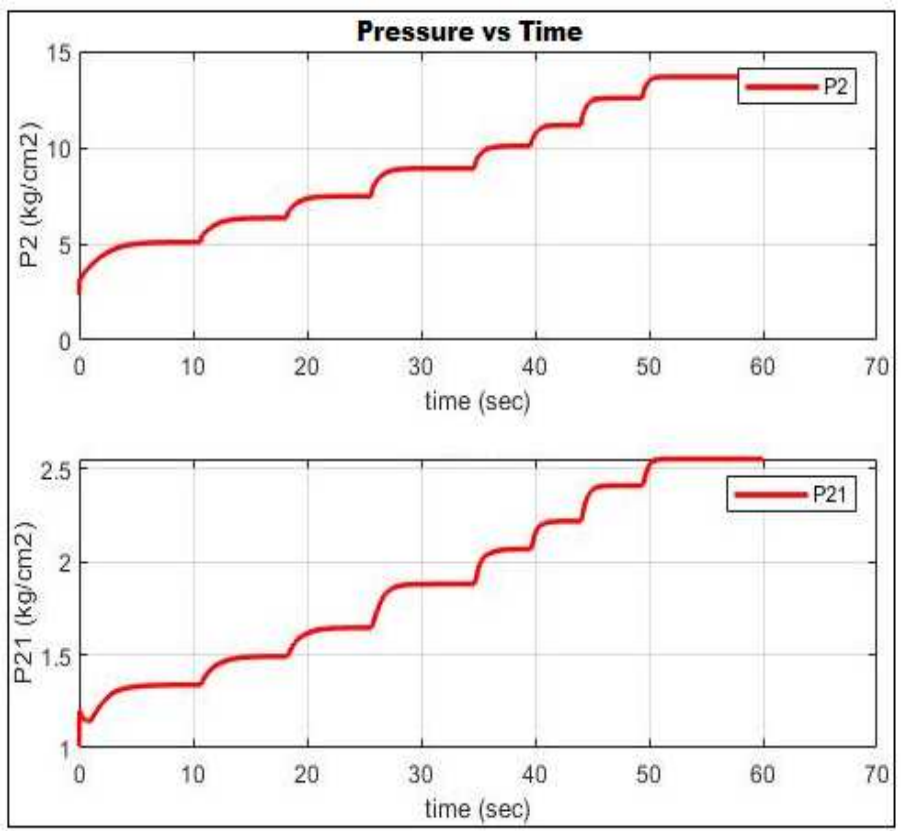

Figure 6: Pressure vs Time Plot.

Furthermore the key feature that signifies the execution or performance of the engine is its speed. The tip of the blade speed basically establishes maximum pressure ratios where it can be derived by compressor and turbine as well.

Suppose if limited fuel delivery condition is occurring then as a result the speed of turbine will begin to drop below the commanded speed and if speed of turbine drops too low then operation can or may be compromised. The figure plot7 is a graph representing, the speed values obtained by running the engine model against time at various levels. The relation between speed at LPC and speed at HPC of the engine run-process are two major stages in combustion process of engine. These are two speed profiles of the engine. High pressure combustion is the penultimate stage after the low pressure combustion before the exhaust stage in the engine run process.

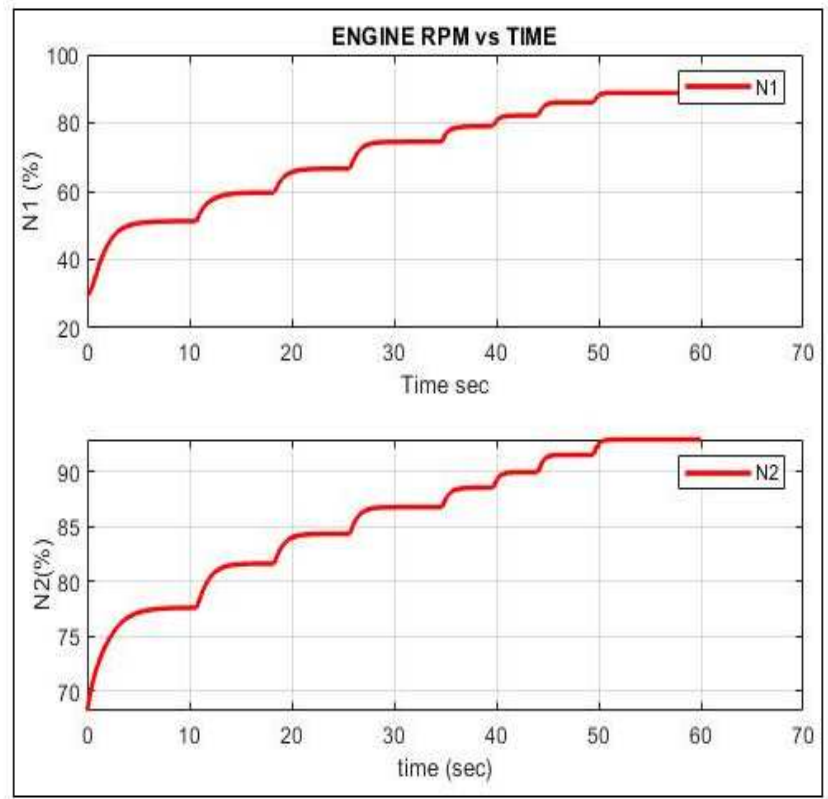

Figure 7: Speed vs Time Plot. 
The first plot shows the relation between N1 (Speed at LPC) and the time while the later depicts the relation between N2 (Speed at HPC) of the engine run-process.High Pressure Combustion (N2) is the penultimate stage after the Low Pressure Combustion (N1) before the exhaust stage in the engine run process.

\section{CONCLUSIONS}

This paper generates all real-time work environment parameters for testing and debugging. The problem addressed is overcome by creating a virtual engine model that is to be connected with ECU for simulation process. However when integrating all individual code blocks of engine model into a single engine model on a Zynq embedded platform, results in a simulation time of 0.2 milli-secondsconsidering all stages from fuel entering through inlet section to nozzle. The given target execution time of an engine model to be run and also overall execution time after simulating an engine model in every stage of engine is achieved. The breakthrough results in execution time less than of the target time. Since the engine model is run on the Zynq processor to validate its performance and quality, the level of resonance of the engine model with the actual gas turbine engine by considering the real-time engine run conditions and parameters within considerable run time is much lesser than the actual engine and it's the major break-through. Hence the input and output parameters of an engine model with respect to time versus fuel flow rate, temperature, pressure and speed is plotted on a MATLAB Simulink respectively.

\section{REFERENCES}

1. Chipperfield, A., \& Fleming, P, “Multiobjective gas turbine engine controller design using genetic algorithms”. IEEE Transactions on Industrial Electronics, 43(5), 583-587. doi:10.1109/41.538616,1996.

2. Xunbo Li, J et.al. "The design and realization of gas turbine engine test visualization, IEEE International Conference on Mechatronics and Automation. doi:10.1109/icma.2008.4798914

3. S M.Montazeri-Gh, H. Yousefpour, and S. Jafari, "Fuzzy Logic Computing for Design of Gas Turbine Engine Fuel Control System” International Conference on Computer and Automation Engineering (ICCAE). doi:10.1109/iccae.2010.5451342, 2010.

4. Patel, Bhavinkumar, and Dattatraya Subhedar."Experimental and Numerical Investigation of Diesel Engine Turbocharger." International Journal of Automobile Engineering Research and Development (IJAuERD) ISSN (P) (2016): $2277-4785$.

5. V. A. Skibin, V. I. Solonin, Yu. A. Temis, Mechanical Engineering. Encyclopedia. V. IV-21. Aircrafts and helicopters. B. 3. Aircraft engines (Mashinostroenie. Entsiklopediya. T. IV-21. Samoletyivertolety.Kn. 3.Aviatsionnyedvigateli). Moscow: Mashinostroenie Publ., 2010. 720 p, 2010.

6. Jack D. Mattingly, “Elements of Gas Turbine Propulsion”, McGraw-Hill Series in Aeronautical and Aerospace Engineering Consulting Editor John D. Anderson, Jr., University of Maryland, 2013.

7. Sharma, Gangaraju Srinivasa, MVS Murali Krishna, and D. N. Reddy."CFD analysis for adaptability of producer gas for power generation in gas turbines." International Journal of Mechanical and Production Engineering Research and Development (IJMPERD) 5.2 (2015): 21-32.

8. Rich Griffin, Silica Xilinx Zynq embedded design tutorial document and Zedboard hardware UG-V2-2, v1.0, July 2014.

9. Zhang, X, "Modeling and simulation of gas turbine engine based on object-oriented approach" 17th International Conference on Control, Automation \&Systems (ICCAS). doi:10.23919/iccas.2017.8204295, 2017. 
10. Ma S. Tan, J, "Modeling and simulation of gas turbine starter and fuel control system", doi:10.23919/chicc.2017.8027674, 2017.

11. User Guide, UG585 ZYNQ-7000 embedded design Technical reference module design tutorial, v1.12.2, July 2018.

12. Joy, Nivin. "Design and Analysis of an Integrated Exhaust Manifold with Turbocharger for Considerable Reduction of Over Heating of Bearing System." International Journal of Mechanical and Production Engineering Research and Development 8.2 (2018): 601-608.

13. Ashok Kumar Gupta, "Design and Implementation of High speed universal asynchronous receiver and transmitter", 7th international conference on signal processing and integrated networks, 2020.

14. Reddy, Sandiri Srikanth, and Manoj Kumar Reddy."Design of Annular Combustion Chamber for a Micro Turbofan Engine. "International Journal of Mechanical and Production Engineering Research and Development (IJMPERD) 9. 6, Dec 2019, 747-754 

